\title{
The complete set of H/ACA snoRNAs that guide rRNA pseudouridylations in Saccharomyces cerevisiae
}

\author{
CLAIRE TORCHET, ${ }^{1}$ GWENAËL BADIS, ${ }^{1,4}$ FRÉDÉRIC DEVAUX, ${ }^{1,2}$ GINNY COSTANZO, ${ }^{1}$ \\ MICHEL WERNER, ${ }^{1,3}$ and ALAIN JACQUIER ${ }^{1}$ \\ ${ }^{1}$ Unité de Génétique des Interactions Macromoléculaires, Institut Pasteur (CNRS-URA 2171), 75724 Paris cedex 15, France \\ ${ }^{2}$ Laboratoire de Génétique Moléculaire, Ecole Normale Supérieure (CNRS-UMR 8541), 75005 Paris, France \\ ${ }^{3}$ Service de Biochimie et de Génétique Moléculaire, Batiment 144, CEA/Saclay, 91191 Gif-sur-Yvette Cedex, France
}

\begin{abstract}
Conversion of uridines into pseudouridines $(\Psi s)$ is the most frequent base modification in ribosomal RNAs (rRNAs). In eukaryotes, the pseudouridylation sites are specified by base-pairing with specific target sequences within $\mathrm{H} / \mathrm{ACA}$ small nucleolar RNAs (snoRNAs). The yeast rRNAs harbor $44 \Psi \mathrm{s}$, but, when this work began, $15 \Psi$ s had completely unknown guide snoRNAs. This suggested that many snoRNAs remained to be discovered. To address this problem and further complete the snoRNA assignment to $\Psi$ sites, we identified the complete set of RNAs associated with the H/ACA snoRNP specific proteins Gar1p and Nhp2p by coupling TAP-tag purifications with genomic DNA microarrays experiments. Surprisingly, while we identified all the previously known H/ACA snoRNAs, we selected only three new snoRNAs. This suggested that most of the missing $\Psi$ guides were present in previously known snoRNAs but had been overlooked. We confirmed this hypothesis by systematically investigating the role of previously known, as well as of the newly identified snoRNAs, in specifying rRNA $\Psi$ sites and found all but one missing guide RNAs. During the completion of this work, another study, based on bioinformatic predictions, also reported the identification of most missing guide RNAs. Altogether, all $\Psi$ guides are now identified and we can tell that, in budding yeast, the $44 \Psi$ s are guided by 28 snoRNAs. Finally, aside from snR30, an atypical small RNA of heterogeneous length and at least one mRNA, all Gar1p and Nhp2p associated RNAs characterized by our work turned out to be snoRNAs involved in rRNA $\Psi$ specification.
\end{abstract}

Keywords: box H-ACA snoRNA; noncoding RNA; pseudouridine; microarrays; Saccharomyces cerevisiae

\section{INTRODUCTION}

Biogenesis of functional rRNAs includes the posttranscriptional covalent modification of many carefully selected nucleotides. These modifications are essentially of two types: methylation of the ribose at the $2^{\prime}$-hydroxyl group or conversion of uridine into pseudouridine $(\Psi)$. These modified nucleotides are exclusively located within the most conserved regions of mature rRNAs and are mostly clustered in and around functional sites (Decatur and Fournier 2002). It was also noticed that the number of modifications increases with evolution (Ofengand et al.

Present address: ${ }^{4}$ Banting and Best Department of Medical Research, University of Toronto, 112 College Street, Toronto, ON M5G 1L6, Canada. Reprint requests to: Alain Jacquier, Unité de Génétique des Interactions Macromoléculaires, Institut Pasteur (CNRS-URA 2171), 28 rue du Dr. Roux, F-75724 Paris cedex 15, France; e-mail: jacquier@pasteur.fr; fax: 33-1-4568 8790

Article and publication are at http://www.rnajournal.org/cgi/doi/ 10.1261/rna.2100905.
1995; Ofengand and Bakin 1997). However, the precise function of these modifications is still unclear. They may contribute to fine-tune rRNA folding and interactions with ribosomal proteins, thereby modulating the biogenesis of the ribosome and enhancing its activity (Maden 1990; Ofengand et al. 1995). Each type of eukaryotic rRNA modifications is directed by a distinct family of guide small nucleolar RNAs (snoRNAs). Ribose methylations are guided by box C/D snoRNAs, whereas pseudouridylations are guided by box H/ACA snoRNAs (for reviews, see Bachellerie et al. 2002; Kiss 2002).

Box H/ACA snoRNAs consist of two irregular stem-loops containing an internal bulge, called the "pseudouridylation pocket." The stem-loops are linked by a single-stranded hinge region that contains the conserved $\mathrm{H}$ box (ANANNA) and are followed by a single-stranded tail containing the ACA box found $3 \mathrm{nt}$ away from the $3^{\prime}$ end (Balakin et al. 1996; Ganot et al. 1997b). The pseudouridylation guide snoRNAs select the substrate uridines by forming two short (3-10 bp) duplexes with rRNA that flank the target uridine 
(Ganot et al. 1997b). The two rRNA recognition motifs occupy the opposite strands of the pseudouridylation pocket. With one guide sequence in each pseudouridylation pocket, one H/ACA snoRNA can potentially specify two $\Psi$ sites.

When we initiated this study, $25 \mathrm{H} / \mathrm{ACA}$ snoRNAs were known in yeast; most of them were originally identified at random, from electrophoretic fractionation of total nuclear small RNAs (Balakin et al. 1996). This number also takes into account four predicted unverified H/ACA snoRNAs, snR161 (Olivas et al. 1997), and RUF1, RUF2, and RUF3 for RNA of unknown function (McCutcheon and Eddy 2003). Sixteen of these 25 H/ACA snoRNAs had been demonstrated to target 20 of the 44 rRNA pseudouridines, whereas nine sites had been predicted to be specified by known snoRNAs (Ganot et al. 1997a; Ni et al. 1997; Ofengand and Fournier 1998; Badis et al. 2003). The remaining $15 \Psi$ sites had not yet been associated with any guide snoRNA. On the other hand, four H/ACA snoRNAs were not associated with any $\Psi$ modification of the rRNA. Additionally, some H/ACA snoRNAs have functions other than rRNA covalent modifications, like snR30 and snR10, which are involved in pre-rRNA cleavages (Tollervey 1987; Morrissey and Tollervey 1993).

All known pseudouridylation guide snoRNAs function in the form of small nucleolar ribonucleoprotein particules (snoRNPs) in which these different site-specific snoRNAs are associated with a set of proteins common to all H/ACA snoRNPs. H/ACA snoRNPs contain four evolutionarily conserved, essential proteins, Cbf5p (dyskerin), Gar1p, Nhp2p, and Nop10p. Cbf5p (Nap57p in mammals) provides the catalytic activity responsible for the snoRNPdirected uridine-to-pseudouridine isomerization reaction. It was previously shown that Garlp has the ability to bind directly snR10 and snR30 H/ACA snoRNAs in vitro (Bagni and Lapeyre 1998), and that Nhp2p is indeed an RNA-binding protein (Henras et al. 2001).

To get a comprehensive view of H/ACA snoRNAs and the rRNA pseudouridylation process, we considered identifying all H/ACA snoRNAs. In order to be as exhaustive and unbiased as possible, we chose an experimental genomic approach consisting of the systematic identification of all RNAs associated with H/ACA snoRNP proteins. Since Nhp2p and Garlp are believed to directly bind all the H/ACA snoRNAs, we chose these proteins as bait for TAP-tag purification. The RNAs coaffinity purified with these baits were used to probe yeast DNA microarrays covering the complete yeast genome, including intergenic regions. Consistent with the approach exhaustively identifying the H/ACA snoRNAs, we found that Nhp2p and Garlp were associated with all 25 previously recognized H/ACA snoRNAs (including RUF1-3). Nevertheless, and quite to our surprise, we found only very few additional RNAs stably associated with Nhp2 and Gar1, only three of which being clearly identified as H/ACA snoRNAs.

Since only three new H/ACA snoRNAs were found, we made the hypothesis that most of the "orphan" $\Psi$ sites were in fact specified by previously known H/ACA snoRNAs that had not been recognized as guiding these positions. This hypothesis was confirmed by experimentally testing, in a systematic way, the involvement in specifying the orphan sites of all new snoRNAs as well as the previously known snoRNAs associated with no or only one $\Psi$ site. This resulted in the experimental determination of 22 guides, including $14 \Psi$ sites that had not been predicted, leaving a single site (LSU-1051) among the $44 \Psi$ s with no assigned guide RNA. Most interestingly, three snoRNAs were found to guide more than two $\Psi$ s and some guide/ target base pairings were found to present evolutionarily conserved mismatches. During the completion of this work, an article was published that reported very similar results but obtained by a totally different, bioinformatic approach (Schattner et al. 2004). In this study, only one additional snoRNA was identified that guides position LSU-1051. Conversely, in comparison to this work, we also identified one additional snoRNA, as well as four new, not previously predicted guides RNAs and experimentally confirmed two additional predictions. Altogether, all $\Psi$ s now have a guide RNA assigned. Finally, we detected one additional atypical RNA of unknown function and at least one mRNA (RPS28A mRNA) stably associated with Nhp2p and Gar1p.

\section{RESULTS}

\section{Identification of RNAs associated with Nhp2p and Gar1p using genomewide analyses}

To identify Nhp2p or Gar1p associated RNAs, we performed a tandem-affinity purification with an Nhp2pTAP or Garlp-TAP tagged strain. Associated RNAs were directly labeled with the Ulysis Nucleic Acid Labeling Kit (see Materials and Methods) with Alexa Fluor 647 dye and competitively hybridized on yeast whole-genome microarrays (see Materials and Methods), competitively with total RNA that had been labeled with Alexa Fluor 546 dye. Experiments have been reproduced three times with three independent immunoprecipitations. The results obtained with both proteins were largely overlapping. For the sake of simplicity, only the Nhp2p example is detailed below. Results for Gar1p and Nhp2p are presented in Supplementary Table S1 (see http://www.pasteur.fr/recherche/unites/ Gim/index.html).

The distribution of the $\log 2$-transformed Alexa 647/Alexa 546 ratios showed a clear peak in the negative values for all experiments (data not shown), indicating that the majority of the RNAs in the cell did not coimmunoprecipitate with Nhp2p in our experiments. Therefore, for microarrays normalization, we fixed the median value of the $\log 2$ transformed ratio distribution at the reference ratio value $(\log 2($ ratio $)=0)$. Then, the normalized ratios represent the enrichment factors $(\mathrm{EF})$ of each RNA in the 
Nhp2p immunoprecipitation versus total RNA, relative to the majority of unprecipitated RNAs. The distribution of the average $\log 2$-transformed EF from the three experiments allowed us to define three groups of RNAs (Fig. 1A). Group 1 represents the majority of RNAs (74\% of all features) that do not coimmunoprecipitate significantly with Nhp2p (EF less than twofold). Group 2 includes a significant number of RNAs (25\%) that were moderately immunoprecipitated with Nhp2p (median EF $\sim 4.5$-fold). Seventy six percent of these RNAs are messenger RNAs that are, for most of them, known to be abundant and stable in the cell. These includes mRNAs from TY elements (12\%) or from genes encoding ribosomal proteins and translation factors (10\%). Nhp2p has been shown to bind RNAs nonspecifically in vitro (Henras et al. 2001). It is therefore reasonable to conclude that these moderate enrichments mainly result from nonspecific interactions during the experimental process.

Group 3 includes 37 features (less than $0.3 \%$ of all features) with very high enrichment factors $(18$-fold $<$ $\mathrm{EF}<200$-fold), which are clearly distinguishable from groups 1 and 2 (Fig. 1A; Supplementary Table S1). All of the 25 previously identified H/ACA snoRNAs are found in this group. These include RUF1, RUF2, and RUF3, which correspond to putative H/ACA snoRNAs identified recently by phylogenetic analysis (McCutcheon and Eddy 2003) and respectively renamed snR84, snR82, and snR83 by Schattner et al. (2004). Four of these snoRNAs were represented twice, by redundant features, while one feature carried both snR31 and snR5 (Supplementary Table S1). Thus, the previously known H/ACA snoRNAs represented 28 of these 37 features. The fact that all the H/ACA snoRNAs identified so far were present within group 3 shows that the RNAs found within this group likely represent a quite exhaustive inventory of the RNAs stably associated with Nhp2p in vivo. Note that C/D box snoRNAs, although as abundant and stable as H/ACA snoRNAs in vivo, are absent from this group, illustrating the specificity of the selection. Among the nine remaining features of group 3, which do not correspond to previously identified snoRNAs, four corresponded to intergenic regions (iYML103c, iYMR246w, iYEL055c, iYBR044c), which do not overlap with any known gene and are also among the 33 most highly enriched RNAs both in Nhp2p and Garlp immunoprecipitation (see Supplementary Table S1). To confirm the presence of potential RNAs interacting specifically with Nhp2p within these intergenic regions, we performed Northern blot analysis (Fig. 1B) on RNA isolated from TEV and TAP eluates of the Nhp2p-TAP tagged strain. Nop1p, a protein specifically associated with C/D snoRNAs, is used as a negative control with a Nop1p-TAP tagged strain. Furthermore, as positive and negative control probes, we used two oligonucleotides complementary to snR191 (an H/ACA snoRNA) and U18 (a box C/D
A

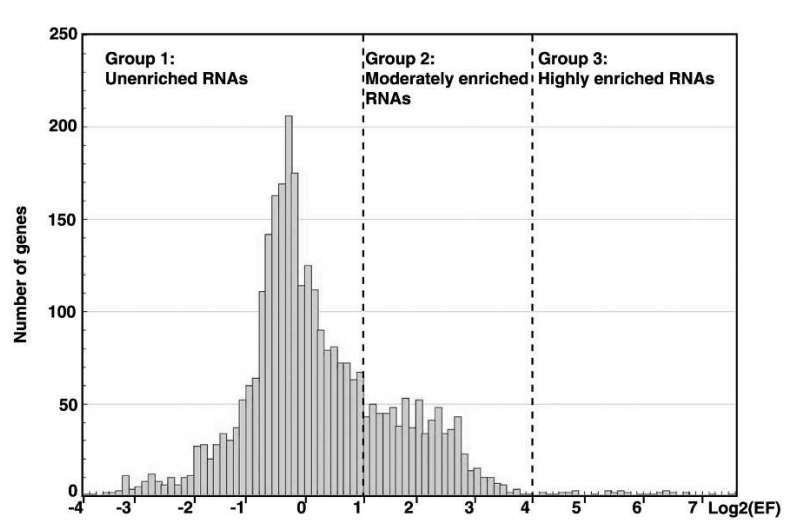

B

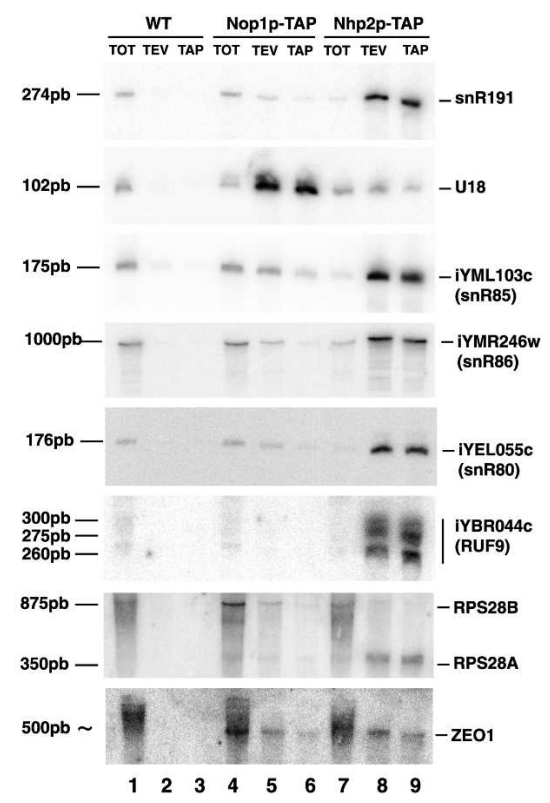

FIGURE 1. RNAs associated with Nhp2p. (A) Distribution of average $\log 2$ transformed enrichment factors (EF) from three independent microarray hybridizations analyzing Nhp2p associated RNAs. The dashed lines indicate the threshold applied for defining three different groups of enrichment. $(B)$ Northern blot analyses. Total RNAs (lanes 1,4,7), RNA isolated from the TEV eluate (lanes 2,5,8) and from the TAP eluate (lanes 3,6,9), from wild-type cell (MGD353-13D) (lanes 1,2,3), from cells carrying Nop1p-TAP (SC0841) (lanes 4,5,6) or Nhp2p-TAP (SC1110) (lanes $7,8,9$ ) were separated by polyacrylamide gel electrophoresis on $5 \%$ gel, blotted, and the blot hybridized probed with specific oligonucleotides (for small RNAs) or random primed PCR products (mRNAs) successively (see Materials and Methods). 
snoRNA), respectively. As expected, snR191 is specifically enriched in the TEV and TAP eluates of Nhp2p strain (Fig. 1B, lanes 8,9), whereas U18 is only enriched in the TEV and TAP eluates of Nop1-TAP strain (Fig. 1B, lanes 5,6). No enrichment was seen in the untagged wild-type strain (Fig. 1B, lanes 2,3).

Analysis with a specific probe within iYML103c reveals an abundant small RNA of $\sim 175$ nucleotides (nt) (Fig. 1B, lanes 8,9), enriched in the TEV and TAP eluates of the Nhp2p-TAP tagged strain compared to the Noplp-TAP strain (Fig. 1B, lanes 5,6). The $5^{\prime}$ end of this small RNA was defined by primer extension analysis (see Supplementary Fig. S1B). The mature small RNA is coded on the Crick strand and begins at nt 67937 of chromosome XIII (coordinates according to the Stanford Saccharomyces Genome Database, SGD, January 13, 2005, [http:// www.yeastgenome.org/]). We estimate that it terminates at position 67763, according to its size and to fit to an H/ACA consensus structure (see Supplementary Fig. S1A). This snoRNA was also recognized by Schattner et al. (2004), who named it snR85.

Likewise, the intergenic region iYEL055c contains a small RNA of $\sim 176 \mathrm{nt}$, specifically enriched in the Nhp2p-TAP strain (Fig. 1B, lanes 8,9). The mature $5^{\prime}$ end of this small RNA, as defined by primer extension (Supplementary Fig. S1D) begins at nt 52318 of the chromosome $\mathrm{V}$ and terminates at position 52143 according to its size (coordinates according to SGD, January 13, 2005) and to fit to an H/ACA consensus structure (Supplementary Fig. S1C). This snoRNA was also recognized by Schattner et al. (2004), who named it snR80.

A specific probe within iYBR044c reveals three bands of $\sim 300,275$, and $260 \mathrm{nt}$, respectively (Fig. 1B). These three species all hybridize with oligonucleotide CT35 (Supplementary Table S3), defining these bands as representing a single transcript of heterogeneous length with a sequence corresponding to the Watson DNA strand. Reverse transcription defined the $5^{\prime}$ ends for these three species as starting at nt $326280 \pm 1 \mathrm{nt}, 326302 \pm 1 \mathrm{nt}$, and $326327 \pm 1 \mathrm{nt}$ of chromosome II (as defined in SGD on March 10, 2005; see Supplementary Fig. S2A). A potential structure, reminiscent of an H/ACA structure and determined with the help of the Mfold program (Walter et al. 1994) is presented in Supplementary Figure S2B for the smallest version of this RNA. No phenotype could be associated with the deletion of this new Nhp2p and Garlp associated RNA and no function could be attributed to it. According to the nomenclature of McCutcheon and Eddy (2003), we propose to name it RUF9 for RNA of unknown function 9. This RNA was not characterized further.

A specific probe within iYMR246w reveals an unusually long RNA of $1000 \mathrm{nt}$ (Fig. 1B, lanes 8,9), enriched in the TEV and TAP eluates of the Nhp2p-TAP tagged strain. All previously known H/ACA snoRNAs are between 161 and $608 \mathrm{nt}$. A more detailed analysis of this atypical new snoRNA, presented below, shows that it exhibits characteristic features of H/ACA snoRNAs and we named it snR86.
Finally, group 3 included four additional features corresponding to RNAs transcribed from the RPS28A, ZEO1, and FAR1 ORFs, respectively, and a Ty element. For the RPS28A mRNA (Fig. 1B), we verified by Northern blot that the positive signal detected on the microarray indeed represented the specific enrichment of the corresponding polyadenylated mRNA in the Nhp2p and Garlp copurifications (Fig. 1B; data not shown; see Discussion). For the ZEO1, although the mRNA was clearly enriched in the Nhp2p copurification, this enrichment appeared less specific for the H/ACA proteins since a significant enrichment was also observed with the Noplp copurification (see Fig. 1B). Likewise, Northern blot analysis showed that the FAR1 mRNA was only very weakly enriched by the Nhp2p copurification and this enrichment was not specific as it was similar in the Nop1p copurification (data not shown). Note that these mRNAs exhibit among the weakest EF in group 3 (between 20 - and 30-fold). We thus conclude that the RPS28A mRNA is the only mRNA specifically associated with the H/ACA specific proteins Nhp2p and Garlp.

\section{SnR86, an atypical H/ACA snoRNA with an unexpected structure}

In order to more precisely characterize snR86, we determined the $5^{\prime}$ end of this RNA by primer extension analysis (data not shown). We concluded that the mature RNA begins at nt 763112 on the Crick strand of the chromosome XIII. Taking into account the $\sim 1000$-nt apparent size observed by Northern blot, we applied this region to comparative sequence analysis using the corresponding intergenic region of Candida glabrata (Dujon et al. 2004). Matrix alignment of both regions using the DNA strider program (Marck 1988) revealed that the primary sequence is not conserved between C. glabrata and S. cerevisiae, except in three short sequences, the longest being located at the $3^{\prime}$ end of the region $(1,2$, and 3 in Fig. 2A). However, despite the divergence of the primary sequences, the RNA secondary structures predicted by the Mfold program (Walter et al. 1994) for C. glabrata and S. cerevisiae were strikingly similar (Fig. 2B,C). These predictions suggest an unexpected structure for a box H/ACA snoRNA, with an unusually long $\mathrm{H}$ box stem structure with a terminal fork, the tips of which precisely correspond to the short homologies 1 and 2 as indicated in Figure 2B-D, while homology 3 corresponded to a sequence with a potential structure typical of the ACA stem-loop. Taking into account the apparent length of the RNA on Northern blot and the location of the ACA box, we concluded that the RNA $3^{\prime}$ end should be located at $3 \mathrm{nt}$ after the ACA box, which corresponds to nt 762109 of the chromosome XIII.

To test whether snR86 functions as guide RNA for rRNA pseudouridylation, we constructed a strain deleted for snR86. Total RNA was isolated from wild-type and deleted strains and the presence of ribosomal $\Psi$ s was tested by the 
A

S. cerevisiae sequence

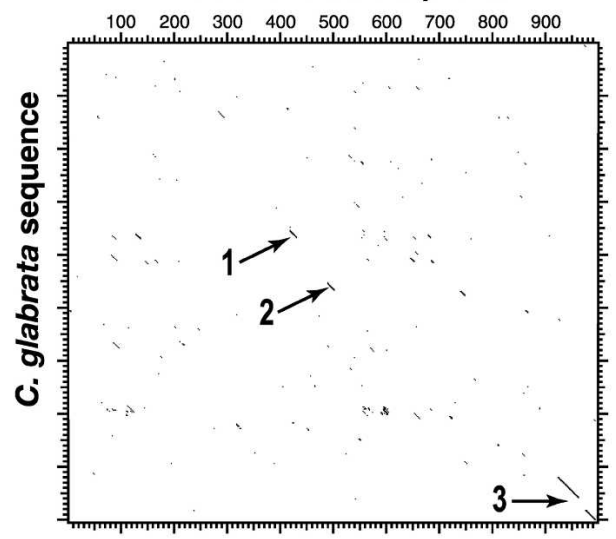

B S. cerevisiae sequence fold

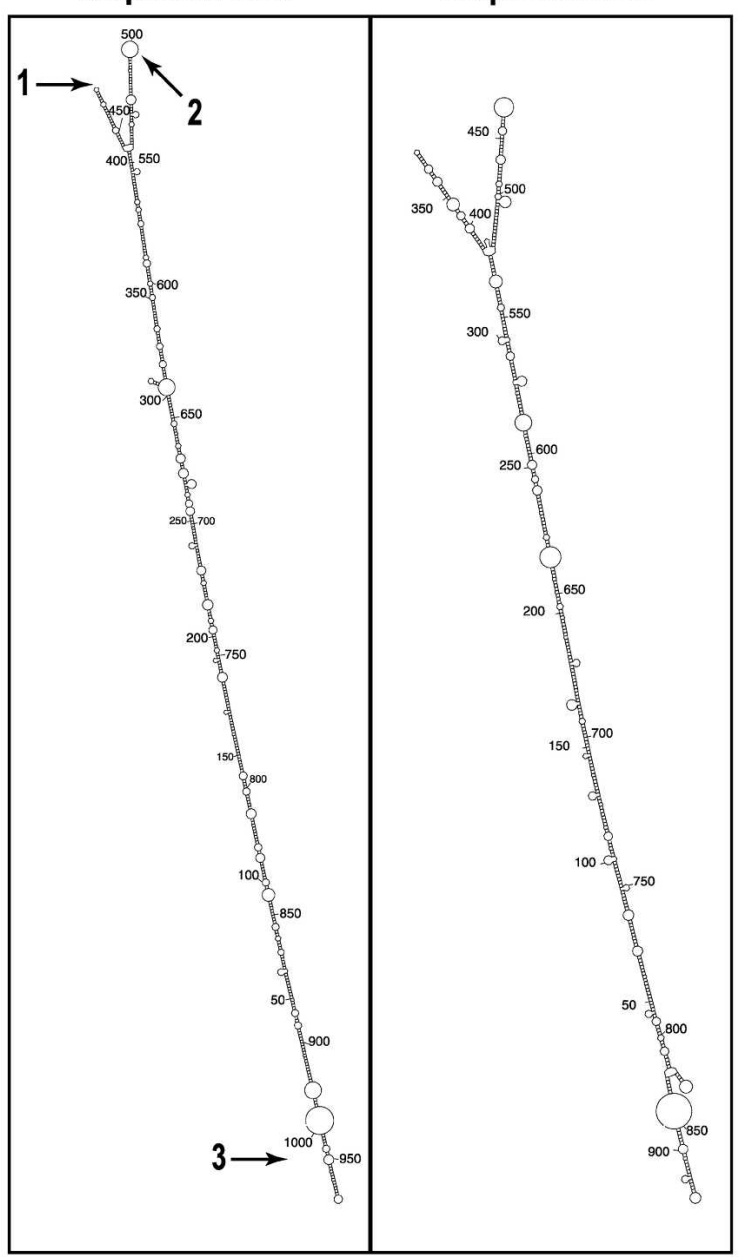

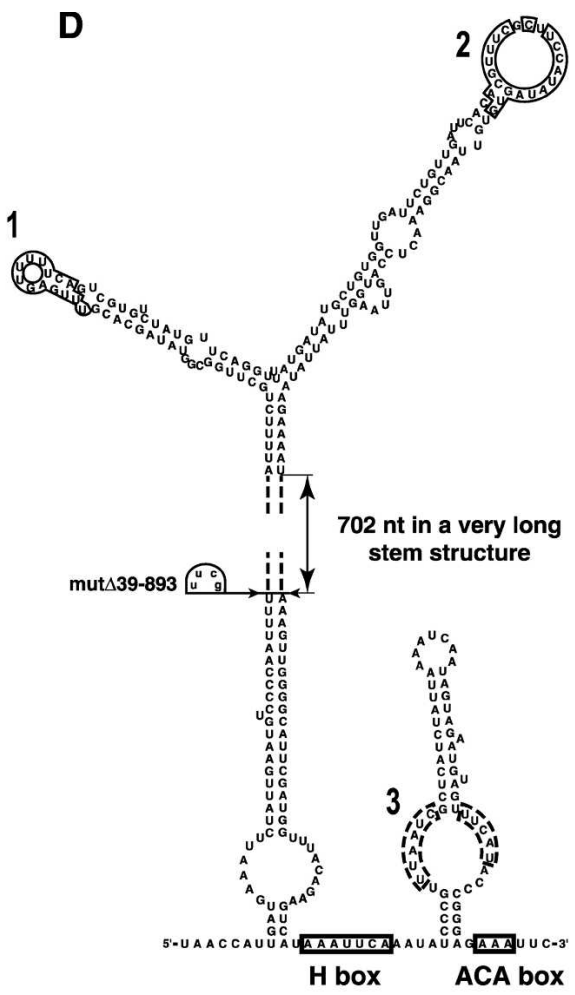

$\mathbf{E}$

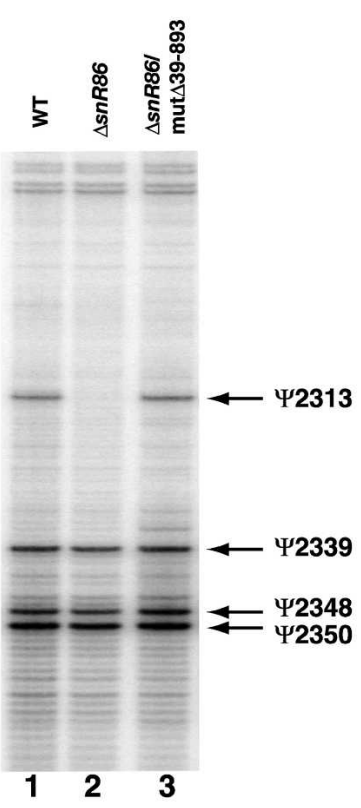

FIGURE 2. Characterization of iYMR246w/snR86. (A) DNA Strider (Marck 1988) matrix alignment of S. cerevisiae snR86 and the corresponding region in C. glabrata. $(B, C)$ Secondary structure of snR86. SnR86 secondary structure predicted using the Mfold program for S. cerevisiae or C. glabrata snR86 sequences ( $B$ and $C$, repectively). $(D)$ Detail of the conserved elements in snR86 structure. Boxes represent the conserved loops between both species. The numbers 1, 2, and 3 correspond to the conserved sequences pointed to in $A$ and $B$. Region 3 corresponds to the H/ACA part of the snoRNA; the recognition loops of the ACA box are indicated with a dotted line. The structure of the mutant mut $\Delta 39-893$ is indicated. (E) Primer extension mapping of pseudouridine residues of LSU rRNA. Total RNAs obtained from wild-type strain (BMA64) (lane 1), $\Delta$ snR86 (LMA462) (lane 2), and $\Delta$ snR86/mut $\Delta 39 / 893$ (lane 3) were traited by the CMC-primer extension method (see Materials and Methods). To map $\Psi 2350, \Psi 2348, \Psi 2339$, and $\Psi 2313$, $5^{\prime}$-end-labeled oligonucleotide primer GB132 (see Supplementary Table S3) was used. The extension products were separated on a $6 \%$ sequencing gel. Positions of pseudouridine residues are indicated. 
CMC-primer extension method. This revealed that, in the $\Delta$ snR86 strain, position LSU-2313 was unmodified, in contrast to wild-type rRNA (Fig. 2E, lane 2). We concluded that depletion of snR86 specifically inhibits $\Psi$ of $25 \mathrm{~S}$ rRNA at position U2313. The putative base-pairing interaction between LSU 2313 and snR86 is shown in Figure 4D, below. Mapping all known pseudouridylation sites in the rRNA obtained from the $\Delta$ snR86 strain showed that no other $\Psi$ was affected by the absence of snR86 (data not shown). To test the importance of the long stem structure of snR86, two independent internal deletions, $\Delta 39-893$ and $\Delta 8-862$, were constructed (Fig. 2D). The resulting p36-snR86- $\Delta 39-893$ RNA accumulated efficiently when expressed in the $\Delta$ snR86 strain (data not shown) and rescued the pseudouridylation of U2313 in the 25S rRNA (Fig. 2E, lane 3). We concluded that the deleted sequence is not essential for the stability of snR86 snoRNA or its function in guiding $\Psi$ at position LSU-2313. Moreover, the p36-snR86- $\Delta 8-862$ construction is not accumulated when expressed in the $\Delta$ snR86 strain (data not shown). Actually, a part of the $5^{\prime}$-terminal helical stem is missing in this construction and as was previously shown for other H/ACA snoRNAs (Bortolin et al. 1999), this motif as well as the $3^{\prime}$-terminal helical stem are essential for accumulation of H/ACA snoRNAs.

In conclusion, snR86 appears to have a canonical H/ACA snoRNA structure, except that the H-box is associated with an unusually long hairpin conserved in structure, but the function of which remains elusive.

\section{Forty-four pseudouridine residues are modified by 20 height box H/ACA snoRNAs in yeast}

Fully modified yeast rRNA contains $43 \Psi$ residues, 13 in the small subunit rRNA (SSU) and 30 in the large subunit (LSU) rRNA (Bakin et al. 1994; Bakin and Ofengand 1995). A known modified base corresponding to the hypermodified 1-methyl-3-(3-amino-3-carboxylpropyl) pseudouridine $\left(\mathrm{m}^{1} \mathrm{acp}^{3} \Psi\right)$ is also present in the SSU rRNA at position 1189 (Bakin and Ofengand 1995). Previous analyses have shown that 16 snoRNAs function as guide RNAs in 20 of these modification (Table 1; Ganot et al. 1997a; $\mathrm{Ni}$ et al. 1997; Ofengand and Fournier 1998; Badis et al. 2003). Potential base-pairing interactions between box H/ACA snoRNAs and rRNAs had been predicted for nine $\Psi$ modifications (Table 1; Ganot et al. 1997a; Ofengand and Fournier 1998; McCutcheon and Eddy 2003). Finally, 15 modified residues did not have snoRNA guides associated (Table 1). In order to confirm predictions and to identify the snoRNAs required for formation of $\Psi$ at orphan positions, we examined the patterns of $\Psi$ rRNA modification in strains deleted for each of the 22 snoRNA encoding genes. SnR5, snR8, snR10, snR30, snR36, and snR191 were not included in our experiments since these snoRNAs have been fully analyzed in previous studies
TABLE 1. Complete set of H/ACA snoRNA guide for rRNA $\Psi$ sites

\begin{tabular}{|c|c|c|c|}
\hline Target site & snoRNA & Predicted & Exp. verified \\
\hline SSU-106 & snR44 & a & b, this work \\
\hline SSU-120 & snR49 & & ${ }^{b}$, this work \\
\hline SSU-211 & snR49 & & b, this work \\
\hline SSU-302 & SnR49 & & b, this work \\
\hline SSU-466 & snR189 & a & ${ }^{b}$, this work \\
\hline SSU-632 & snR161 & & ${ }^{b}$, this work \\
\hline SSU-759 & snR80 & & This work \\
\hline SSU-766 & snR161 & & ${ }^{b}$, this work \\
\hline SSU-1000 & snR31 & & c \\
\hline SSU-1179 & snR85 & b & This work \\
\hline SSU-1185 & snR36 & & a \\
\hline SSU-1189 & snR35 & a & Still unverified \\
\hline SSU-1289 & RUF3/snR83 & & b, this work \\
\hline SSU-1414 & RUF3/snR83 & & This work \\
\hline LSU-775 & snR80 & & ${ }^{b}$, this work \\
\hline LSU-959 & snR8 & & c \\
\hline LSU-965 & snR43 & d & This work \\
\hline LSU-985 & snR8 & & c \\
\hline LSU-989 & snR49 & a & ${ }^{b}$, this work \\
\hline LSU-1003 & snR5 & & a \\
\hline LSU-1041 & snR33 & & c \\
\hline LSU-1051 & snR81 & & b \\
\hline LSU-1055 & snR44 & a & ${ }^{b}$, this work \\
\hline LSU-1109 & RUF2/snR82 & & This work \\
\hline LSU-1123 & snR5 & & $\mathrm{a}$ \\
\hline LSU-2128 & snR3 & & ${ }^{b}$, this work \\
\hline LSU-2132 & snR3 & c & $\mathrm{b}^{\prime}$, this work \\
\hline LSU-2190 & snR32 & & c \\
\hline LSU-2257 & snR191 & & e \\
\hline LSU-2259 & snR191 & & e \\
\hline LSU-2263 & snR3 & & c \\
\hline LSU-2265 & RUF1/snR84 & & ${ }^{b}$, this work \\
\hline LSU-2313 & snR86 & & This work \\
\hline LSU-2339 & snR9 & & \\
\hline LSU-2348 & RUF2/snR82 & $f$ & b, this work \\
\hline LSU-2350 & RUF2/snR82 & & ${ }^{b}$, this work \\
\hline LSU-2415 & snR11 & & ${ }^{d}$, this work \\
\hline LSU-2730 & snR189 & & ${ }^{d}$, this work \\
\hline LSU-2822 & snR34 & & ${ }^{d}$, this work \\
\hline LSU-2861 & snR46 & & c \\
\hline LSU-2876 & snR34 & & c \\
\hline LSU-2919 & snR10 & & c \\
\hline LSU-2940 & snR37 & & a \\
\hline LSU-2971 & snR42 & & c \\
\hline
\end{tabular}

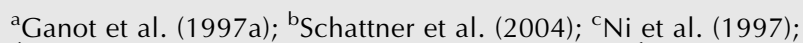
dOfengand and Fournier (1998); ${ }^{\mathrm{e} B a d i s}$ et al. (2003); ${ }^{\mathrm{f}}$ McCutcheon and Eddy (2003).

(Morrissey and Tollervey 1993; Ganot et al. 1997a; Ni et al. 1997; Badis et al. 2003). Of these 22 snoRNAs, 11 were known to modify one or two residues (snR3, snR9, snR11, snR31, snR32, snR33, snR34, snR37, snR42, snR46, and snR189) (Ni et al. 1997; Ganot et al. 1997a; Ofengand and Fournier 1998), nine were predicted to guide one or two modifications (snR3, snR34, snR11, snR35, snR43, snR44, snR49, snR189, and RUF2) (Ganot et al. 1997a; McCutcheon and Eddy 2003) and seven were not associated with any $\Psi$ site: snR43, snR161, RUF1, RUF3 
(Ni et al. 1997; Olivas et al. 1997; McCutcheon and Eddy 2003), snR85, and snR80, which were found in our microarrays experiments and recently identified by bioinformatic analyses (Schattner et al. 2004), and snR86, which was identified in this work (see previous paragraph).

To test whether these 22 snoRNAs are required for the formation of $\Psi$ at positions not yet tested, we constructed 22 strains, each containing one snoRNA gene disruption (Supplementary Table S2 and Materials and Methods). Total RNAs were isolated from two independent clones for each strain (except for $\Delta$ snR80 [LMA459] and $\Delta$ snR43 [LMA461] for which only one clone was analyzed) and the distribution of ribosomal pseudouridines was tested by a primer extension assay after treating the RNA with $\mathrm{N}$-cyclohexyl$N^{\prime}-\beta$-(4-methylmorpholinium)-ethylcarbodiimide $p$-tosylate (CMC) (see Materials and Methods).

Ten sites had been predicted to be specified by nine snoRNAs (Ganot et al. 1997a; Ofengand and Fournier 1998; McCutcheon and Eddy 2003; Schattner et al. 2004). Six of these positions were independently verified experimentally in this study (Table 1; Supplementary Fig. S3) and by Schattner et al. (2004). Moreover, our experiments confirmed the predictions made for two additional positions (Ofengand and Fournier 1998; Schattner et al. 2004): Modifications of residues SSU-1179 and LSU-965 are indeed guided by snR85 and snR43, respectively (Table 1; Fig. 3). In contrast, snR11 is not involved in pseudouridylation of LSU-2128 residue as predicited by Ganot et al. (1997a) (data not shown); instead snR11 guides the LSU2415 modification (Supplementary Fig. S3; Schattner et al. 2004), while modification of LSU-2128 is guided by snR3 (Supplementary Fig. S3). The prediction that snR35 guides the pseudouridylation of the base $m^{1} a^{3} \Psi$ at position SSU-1189 remains unverified as the strong reverse transcriptase stop observed at this position (Bakin and Ofengand 1995) is not influenced by deletion of snR35 (data not shown), probably as a result of the hypermodification of the residue (Raue et al. 1988).

Out of the 15 remaining orphan $\Psi$ s, 11 were associated with guide snoRNAs by Schattner et al. (2004). We independently identified experimentally 10 out of these 11 guides (Table 1; Supplementary Fig. S3), the only one that we missed being snR81, absent from our array results, and which guides the modification of LSU1051 (Schattner et al. 2004). Finally, we assigned guide snoRNAs for the four remaining orphan positions, SSU759, SSU-1414, LSU1109, and LSU2313 (snR80, RUF3, RUF2, and snR86, respectively; Table 1; Figs. 2E, 3).

\section{DISCUSSION}

In yeast, $44 \Psi$ sites have been identified in rRNAs and, at the time we initiated this study, only 20 of these positions had been linked to a guide H/ACA snoRNA. On the other hand, $25 \mathrm{H} / \mathrm{ACA}$ snoRNAs potentially guiding rRNA pseudouridylation had been identified, but only 16 were experimentally tested for their role in pseudouridylation. The work described in this article first aimed at completing and clarifying our knowledge of rRNA pseudouridylation and of the composition and roles of the H/ACA snoRNA family in yeast. The first possibility that we investigated was that many new H/ACA snoRNAs remained to be discovered in the yeast genome. In addition, the important question of whether, in yeast, all H/ACA snoRNAs were involved in rRNA maturation and modification or whether there would be additional H/ACA snoRNAs involved in additional functions had not yet been directly addressed. Through a combination of protein immunoprecipitation and DNA microarray analyses, we identified all previously identified H/ACA snoRNAs, but found only three additional snoRNAs associated with the yeast Nhp2p and Garlp H/ACA snoRNP proteins and clearly involved in pseudouridylation (snR80, snR85, and snR86). Among these, the snoRNA that we called snR86 presented a striking, atypical structure. SnR86 is unusually long (1000 nt) and presents a spectacular long stem structure. As of now, snR86 is a unique case in the H/ACA family. The functional meaning of its atypical structure remains elusive since, although the structure of the long forked stem, as well as the sequences of the apical loops, are conserved between S. cerevisiae and C. glabrata (Fig. 2A-D), a deletion of most of the stem did not affect snR86-guided pseudouridylation of position LSU-2313 (Fig. 2D,E).

The identification of only three new snoRNAs involved in rRNA pseudouridylation suggested that some H/ACA snoRNAs previously identified but not fully characterized were responsible for the pseudouridylation of most of the orphan rRNA $\Psi$ sites. We therefore systematically tested the known and newly described H/ACA snoRNAs for their role in rRNA $\Psi$ modifications. This allowed us to experimentally verify nine predicted guides and identify guides for 14 out of the 15 remaining orphan $\Psi$ residues (see Table 1). During the completion of this work, an article was published that reported the identification of guide snoRNAs for many orphan rRNA $\Psi$ residues using bioinformatic predictions followed by experimental validations (Schattner et al. 2004). The completely different approaches used in Schattner's article and the present study make these two works remarkably complementary. Indeed, while most results are in common, we identified an additional H/ACA snoRNA, snR86. This snoRNA escaped the bioinformatic search of Schattner et al. (2004), most likely because of its very unusual structure. Conversely, Schattner and colleagues identified one H/ACA snoRNA, snR81, that escaped our screen. Northern blot experiments showed that snR81 was efficiently immunoprecipitated in our Nhp2p and Garlp TAP-TAG fractions (data not shown); the corresponding signal was nevertheless absent in our microarray experiments, possibly due to technical problem with the corresponding intergenic probe spotted on the arrays. Aside from $\Psi$ at position LSU-1051, which is 
A

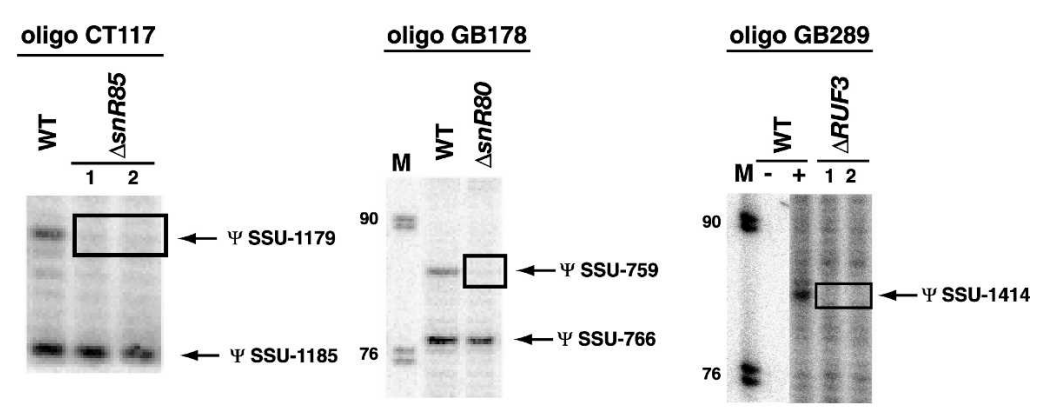

B

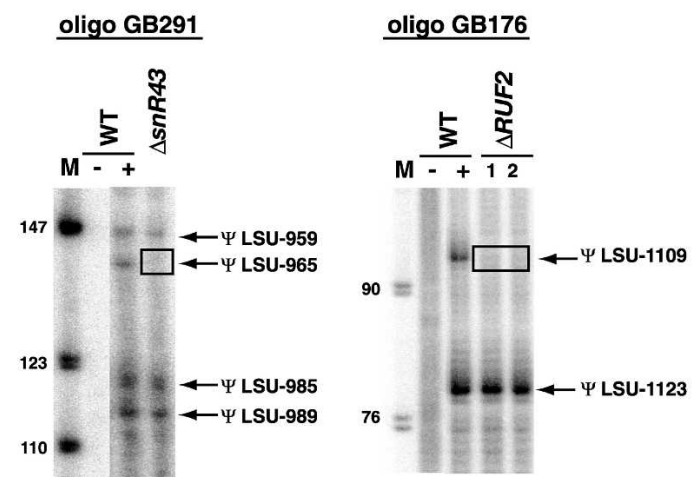

FIGURE 3. Primer extension analyses of small and large subunit rRNA pseudouridylations. The presence of $\Psi$ s was monitored by the CMC-primer extension method (see Materials and Methods). Results are shown for five strains, each depleted of a different snoRNA with two independent clones, 1 and 2, except for $\Delta \operatorname{snR} 43, \Delta \operatorname{snR} 80$ with one clone and for the wild-type strain, BY4742, or BMA64 in the case of $\Delta$ snR43. Only the rRNA regions affected are shown. When appropriate, CMC treatment is indicated by - (untreated) and + (treated) for wild-type RNAs. Positions of pseudouridine residues are indicated. Lanes $M$ represent size markers. $(A)$ $\Psi$ s within SSU rRNA. (B) $\Psi$ s within LSU rRNA. The oligonucleotides used are listed in Supplementary Table S3.

specified by snR81, our experiments independently identified or verified all guide snoRNAs found by Schattner et al. (2004). In addition, we found that snR80, RUF3/snR83, RUF2/snR82, and snR86 guide for $\Psi$ s at positions SSU759, SSU-1414, LSU-1109, and LSU-2313, respectively (see Table 1; Fig. 2-4). Interestingly, it was suggested by Schattner and colleagues that positions SSU-759 and LSU-2313 could be guided by multiple, redundant snoRNAs (Schattner et al. 2004). Our work clearly shows that this is not the case and that, in fact, all $\Psi$ s are specified by a single snoRNA. Likewise, the $\Psi$ at position LSU-1109 was predicted by Schattner and colleagues to be specified by snR30 (which exhibits a convincing guide sequence for this position), but we experimentally found this position to be, in fact, modified by RUF2/snR82 (see Figs. 3, 4). Schattner and coworkers had also found that at least two snoRNAs, snR3 and snR49, were able to guide more than two (three and four, respectively) Is (Schattner et al. 2004). Our work confirmed this finding and extended this observation to RUF2/snR82, which we also found involved in $\Psi$ modification at three distinct sites (see Table 1; Figs. 3, 4). Figure $4 \mathrm{E}$ presents a hypothetical structural rearrangement within RUF2/snR82 that might explain how the $\Psi \mathrm{s}$ at positions LSU-2348 and LSU-2350 could be specified by the same guide sequence. Finally, our work confirmed the yet unverified predictions (Ofengand and Fournier 1998; Schattner et al. 2004) that $\Psi$ s at positions SSU-1179 and LSU-965 are specified by snR85 and snR43, respectively. Importantly, with our work, all $\Psi$ s have an assigned guide snoRNA (all, but snR35 potentially guiding the hypermodified position LSU-1189, were experimentally verified) and we can thus now state that the 44 rRNA $\Psi$ s are guided by 28 distinct H/ACA snoRNAs. Thus, in contrast to the situation observed in bacteria, no rRNA $\Psi$ is modified by a snoRNA independent enzyme. It is also worth noting that none of the 22 snoRNA deletions (see Supplementary Table S2) gave rise to an obvious growth defect at $30^{\circ} \mathrm{C}$ on complete medium.

Another important conclusion that can be drawn from our work is that there are probably no, or only a very few, additional H/ACA snoRNAs not involved in rRNA modification or maturation (the atypical RUF9 RNA, in the hypothesis that its structures would indeed conform to genuine H/ACA specific structures, would be, with snR30, which is involved in rRNA maturation, the only H/ACA snoRNA not guiding a rRNA $\Psi)$. This result is very significant because, aside from snR81, our approach identified all the snoRNAs guiding the rRNA Is. Finally, the RPS28A appears to be the only mRNA specifically associated with H/ACA proteins. Several hypotheses can explain the presence of this specific mRNA within the Nhp2p and Garlp immunoprecipitates. First, it could represent an mRNA containing $\Psi$ s incorporated within the nucleolus by H/ACA snoRNPs. The pseudouridylation of messenger RNAs has been mentioned in the literature (Kiss 2002), although it has never been demonstrated. In a second hypothesis, this mRNA would be captured by H/ACA snRNP proteins to be sequestered within the nucleolus for regulation purposes. In support of this alternative model is the finding that $R P S 28 B$, the second copy of the gene encoding Rps $28 \mathrm{p}$, is itself regulated posttranscriptionally, although by a completely different mechanism (Badis et al. 2004). Furthermore, the use of the Mfold program (Walter et al. 1994) suggests that this mRNA 
A

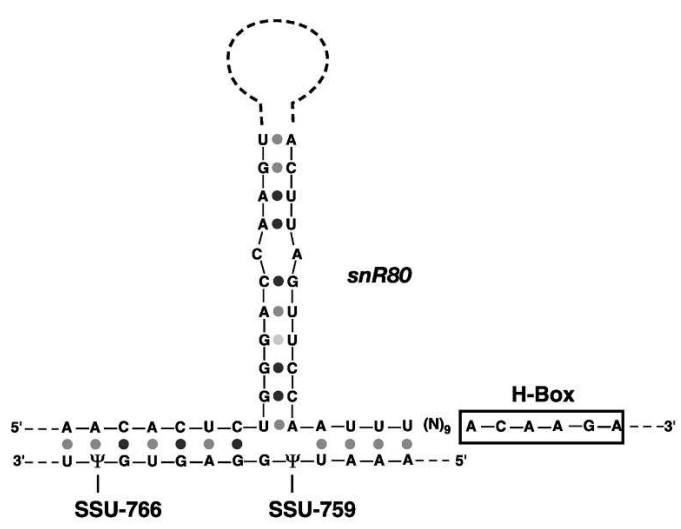

C

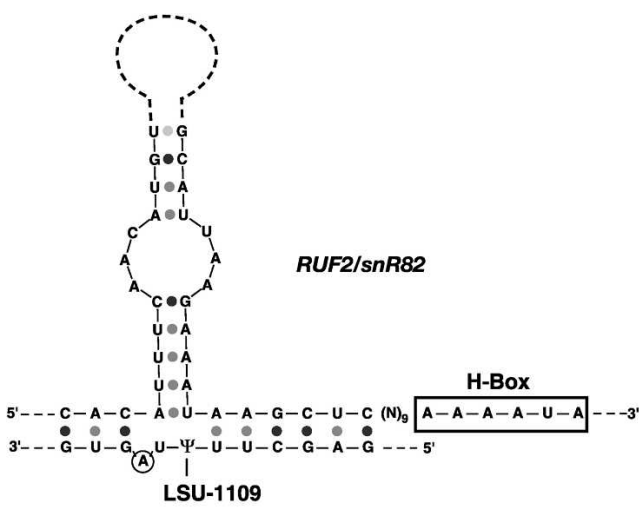

B

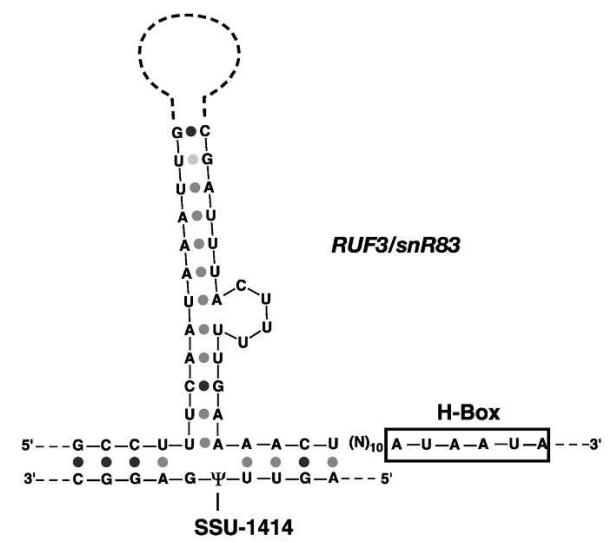

D

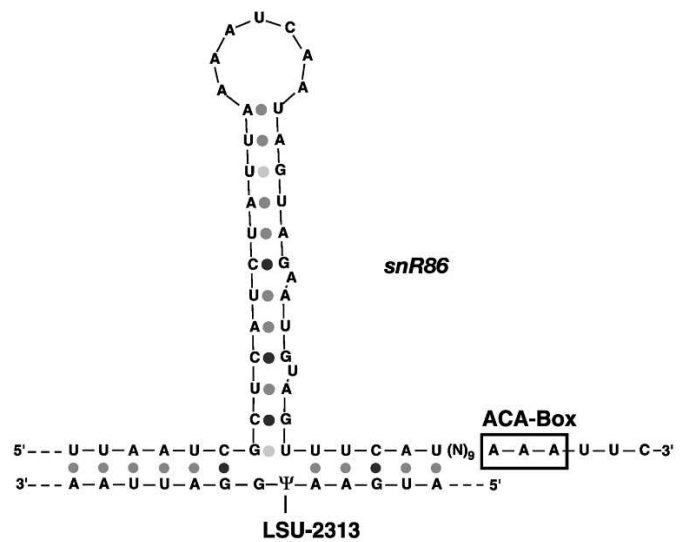

$\mathbf{E}$

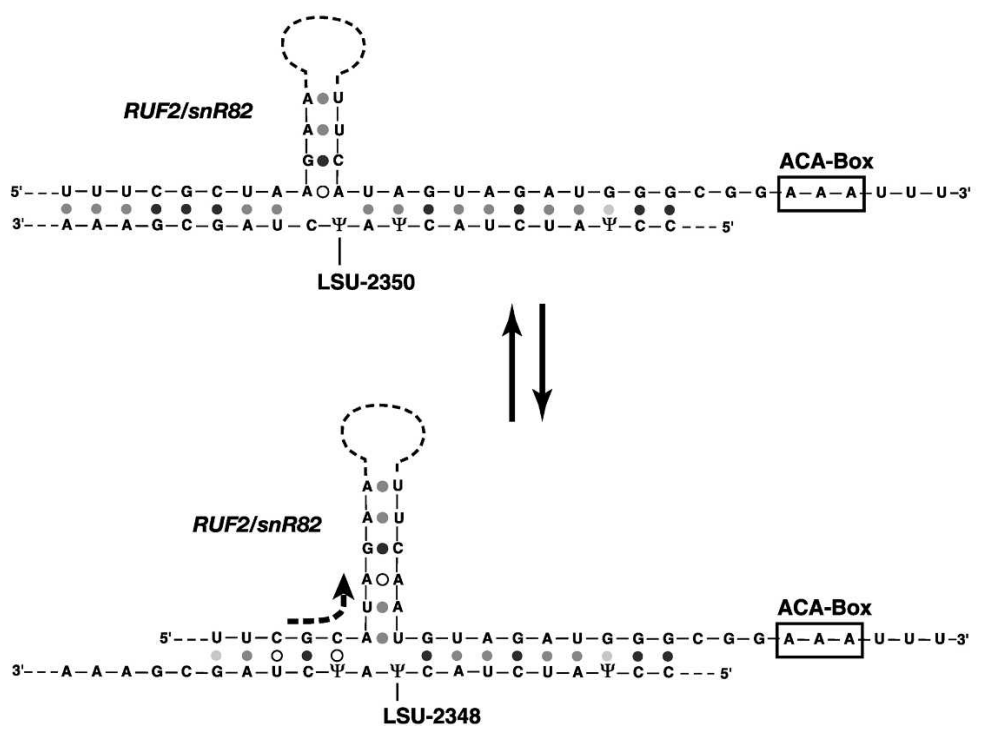

FIGURE 4. Potential base-pairing interactions between $\Psi$ target regions in rRNA and potential guide sequences within snoRNAs. $(A)$ Potential snR80 guiding interaction with the SSU-759 site. (B) Potential RUF3/snR83 guiding interaction with the SSU-1414 site. (C) Potential RUF2/snR82 guiding interaction with the LSU-1109 site. (D) Potential guiding interaction with the newly identified H/ACA snoRNA snR86 and the LSU-2313 site. (E) Hypothetical structural rearrangement within RUF2/snR82 that could explain how a unique ACA-box associated guide can specify two adjacent $\Psi$ s within the LSU rRNA. 
could indeed adopt an H/ACA structure (see Supplementary Fig. S4). Work is in progress to test these possibilities.

\section{MATERIALS AND METHODS}

\section{Yeast strain}

For a full description of yeast strains used, see Supplementary Table S2. The TAP-tagged Nhp2p strain (SC0841) and the TAPtagged Garlp strain (SC1110) were obtained from Cellzome (Gavin et al. 2002). Genomic integration of the TAP tag at the $3^{\prime}$ end of NOP1 (LMA439) was performed as described previously (Puig et al. 2001) with primers NOP1-A and NOP1-B. Correct integration of the tag was verified by immunoblot analysis of the cell extract (data not shown).

SnoRNA disruption strains were generated by transforming BY4742 with a PCR fragment obtained from plasmid pFA6aKanMX6 by the method of Longtine et al. (1998) with primers snRx-F1 and snRx-R1 (see Supplementary Table S3). Correct integration was confirmed by PCR with primers $s n R x-5^{\prime}$ and snRx $-3^{\prime}$ (see Supplementary Table S3). In each case two strains were selected for analysis. LMA461 and LMA462 were constructed by transforming BMA64 with a PCR fragment obtained from plasmid pFA6a-TRP1 by the same protocol, and correct disruption was confirmed by Northern hybridization using kinase-labeled oligonucleotides CT30 and GB99, respectively (see Supplementary Table S3). One correct strain was selected for analysis.

\section{Oligonucleotides}

Oligonucleotides used in this study are listed in Supplementary Table S3.

\section{Immunoprecipitation and TAP purifications}

Tap purification was performed as described previously (LegerSilvestre et al. 2004), except that $10 \mathrm{mM}$ Ribonucleoside Vanadyl Complex (Biolabs) was added to lysis buffer, and $160 \mathrm{U}$ of Rnasin (Invitrogene) were added to the TEV cleavage buffer. Immunoprecipitated RNAs were isolated by phenol/chloroform extraction and ethanol precipitation from a fraction of the TEV eluates (oneforth) as control, and from the TAP eluates, which correspond to the second affinity purification. Total RNAs were isolated from extracts (input) by the same methods.

\section{Microarray and data analysis}

Total RNA $(5 \mu \mathrm{g})$ isolated from extract and three-forths of TAPisolated RNAs were coupled with Alexa fluor 546 or 647, respectively, according to the protocol of the manufacturer (Molecular Probes Ulysis kit), purified with a QIAquick spin column (QIAquick Nucleotide Removal Kit), and hybridized to DNA microarrays representing the whole $S$. cerevisiae genome (including genes and intergenic sequences) (Harismendy et al. 2003). Experiments were reproduced three times with three independent RNA samples. Microarrays were scanned using a Genepix 4000B instrument from Axon. Images were analyzed with the GENEPIX 4.0 software. We excluded artifactual, saturating, and low signal (intensity $<$ background + twofold standard deviation) spots. Observing that the majority of the features ( $\sim 75 \%$ in all experiments) exhibited the same behavior (i.e., low 647/546 ratios), we normalized the results using the median of all ratios. The normalized ratios are then enrichment factors (EF) representing the enrichment of each RNA in the TAP tag immunoprecipitation compared to total RNA (input), relative to the majority of unenriched RNAs. For the final results presented in Figure 1A, we kept only features for which EF were significantly measured in at least two of the three experiments. The complete microarray results for Nhp2p and Gar1p TAP-tag immunoprecipitations are available on request.

\section{Northern hybridization and analysis of $\Psi$ modifications}

Five micrograms of total RNA isolated from extract, one-forth of TEV and TAP-isolated RNA, respectively, were separated on a $5 \%$ acrylamide gel containing $8.3 \mathrm{M}$ urea and electroblotted onto a Hybond- $\mathrm{N}^{+}$nylon membrane, then probed with kinase-labeled oligonucleotides complementary to snR191 (cs9), U18, snR85 (CT33), snR86 (GB99), snR80 (CT124), and RUF9 (CT34). For RPS28 and ZEO1, probes were made by random priming (Nonaprimer kit) of PCR product with oligonucleotides GB171, GB154 and ZEO1-U, ZEO1-L, respectively. Primer extensions were performed as described (Saveanu et al. 2001). Mapping pseudouridines' position on $18 \mathrm{~S}$ and $25 \mathrm{~S}$ rRNA was monitored by the CMCprimer extention method as previously described (Badis et al. 2003). The sequence of oligonucleotides used as probes for mapping ribosomal pseudouridines is indicated in Supplementary Table S3.

\section{Construction of snR86 mutants}

The snR86 gene was amplified by PCR amplification of genomic DNA from BMA64 cells, using primers GB127 and GB128 (see Supplementary Table S3). The resulting DNA fragment was cloned into plasmid pCR-TOPO cloning (Invitrogen). The BamHI-NotI fragment of this plasmid was cloned at the corresponding sites of the pFL36-CII (kindly provided by F. Lacroute, CGM.CNRS. UPR2167, Gif-sur-Yvette) centromeric plasmids yielding plasmid p36-snR86. Mutations of snR86 carrying internal deletion $\Delta 39$ $893 \mathrm{nt}$ and $\Delta 8-862 \mathrm{nt}$ were generated by the "overlap extension PCR" technique (Pogulis et al. 1996) using p36-CII-snR86 as template. In a first step, the sequences on either side of the deletion are amplified by PCR with oligonucleotides (GB35 and CTA25) and (CTA26 and AJ48) for $\Delta 39-893$ and (GB35 and CT14) and (CT13 and AJ48) for $\Delta 8-862$ (see Supplementary Table S3). In a second step, as the two different PCR product contain a region of common sequence, the two overlapping fragments are then fused in a subsequent PCR reaction, using oligonucleotides (GB35 and AJ48). The resulting PCR products were digested by BamH1/Not1 and inserted into the same sites of pFL36-CII, yielding plasmids p36-snR86- $\Delta 39-893$ and p36-snR86- $\Delta 8-862$. The constructs were verified by sequencing.

\section{Computer analysis of snoRNA secondary structures}

Secondary structure analyses were performed with the Mfold program (Walter et al. 1994). 


\section{SUPPLEMENTARY DATA}

Supplementary data are available at the following Web site: http://www.paseteur.fr/recherche/unites/Gim/index.html.

\section{ACKNOWLEDGMENTS}

We are grateful to C. Jacq, who granted us access to microarray facilities at the Ecole Normale Superieure; to the staff of the Service de Genomique Fonctionelle at the CEA-Evry, who printed the whole-genome yeast microarrays; to P. Schattner for the communication of results before publication; and to E. Bray for providing tools for data analysis. We thank all members of the laboratory for help and useful discussions. C.T. was the recipient of a post-doc fellowship from ARC. G.B. was supported by a grant from the European Commission (RNOMICS: QLG2-CT-2001-01554).

Received January 19, 2005; accepted March 18, 2005.

\section{REFERENCES}

Bachellerie, J.P., Cavaille, J., and Huttenhofer, A. 2002. The expanding snoRNA world. Biochimie 84: 775-790.

Badis, G., Fromont-Racine, M., and Jacquier, A. 2003. A snoRNA that guides the two most conserved pseudouridine modifications within rRNA confers a growth advantage in yeast. RNA 9: 771-779.

Badis, G., Saveanu, C., Fromont-Racine, M., and Jacquier, A. 2004. Targeted mRNA degradation by deadenylation-independent decapping. Mol. Cell 15: 5-15.

Bagni, C. and Lapeyre, B. 1998. Garlp binds to the small nucleolar RNAs snR10 and snR30 in vitro through a nontypical RNA binding element. J. Biol. Chem. 273: 10868-10873.

Bakin, A. and Ofengand, J. 1995. Mapping of the 13 pseudouridine residues in Saccharomyces cerevisiae small subunit ribosomal RNA to nucleotide resolution. Nucleic Acids Res. 23: 3290-3294.

Bakin, A., Lane, B.G., and Ofengand, J. 1994. Clustering of pseudouridine residues around the peptidyltransferase center of yeast cytoplasmic and mitochondrial ribosomes. Biochemistry 33: 13475-13483.

Balakin, A.G., Smith, L., and Fournier, M.J. 1996. The RNA world of the nucleolus: Two major families of small RNAs defined by different box elements with related functions. Cell 86: 823-834.

Bortolin, M.L., Ganot, P., and Kiss, T. 1999. Elements essential for accumulation and function of small nucleolar RNAs directing sitespecific pseudouridylation of ribosomal RNAs. EMBO J. 18: 457-469.

Decatur, W.A. and Fournier, M.J. 2002. rRNA modifications and ribosome function. Trends Biochem. Sci. 27: 344-351.

Dujon, B., Sherman, D., Fischer, G., Durrens, P., Casaregola, S., Lafontaine, I., De Montigny, J., Marck, C., Neuveglise, C., Talla, E., et al. 2004. Genome evolution in yeasts. Nature 430: 35-44.

Ganot, P., Bortolin, M.L., and Kiss, T. 1997a. Site-specific pseudouridine formation in preribosomal RNA is guided by small nucleolar RNAs. Cell 89: 799-809.

Ganot, P., Caizergues-Ferrer, M., and Kiss, T. 1997b. The family of box ACA small nucleolar RNAs is defined by an evolutionarily conserved secondary structure and ubiquitous sequence elements essential for RNA accumulation. Genes \& Dev. 11: 941-956.

Gavin, A.C., Bosche, M., Krause, R., Grandi, P., Marzioch, M., Bauer, A., Schultz, J., Rick, J.M., Michon, A.M., Cruciat, C.M., et al. 2002. Functional organization of the yeast proteome by systematic analysis of protein complexes. Nature 415: 141-147.

Harismendy, O., Gendrel, C.G., Soularue, P., Gidrol, X., Sentenac, A., Werner, M., and Lefebvre, O. 2003. Genome-wide location of yeast RNA polymerase III transcription machinery. EMBO J. 22: $4738-4747$.
Henras, A., Dez, C., Noaillac-Depeyre, J., Henry, Y., and CaizerguesFerrer, M. 2001. Accumulation of H/ACA snoRNPs depends on the integrity of the conserved central domain of the RNA-binding protein Nhp2p. Nucleic Acids Res. 29: 2733-2746.

Kiss, T. 2002. Small nucleolar RNAs: An abundant group of noncoding RNAs with diverse cellular functions. Cell 109: 145-148.

Leger-Silvestre, I., Milkereit, P., Ferreira-Cerca, S., Saveanu, C., Rousselle, J.C., Choesmel, V., Guinefoleau, C., Gas, N., and Gleizes, P.E. 2004. The ribosomal protein Rps15p is required for nuclear exit of the $40 \mathrm{~S}$ subunit precursors in yeast. $E M B O \mathrm{~J} .23$ : 2336-2347.

Longtine, M.S., McKenzie 3rd, A., Demarini, D.J., Shah, N.G., Wach, A., Brachat, A., Philippsen, P., and Pringle, J.R. 1998. Additional modules for versatile and economical PCR-based gene deletion and modification in Saccharomyces cerevisiae. Yeast 14: 953-961.

Maden, B.E. 1990. The numerous modified nucleotides in eukaryotic ribosomal RNA. Prog. Nucleic Acid Res. Mol. Biol. 39: 241-303.

Marck, C. 1988. 'DNA Strider': A 'C' program for the fast analysis of DNA and protein sequences on the Apple Macintosh family of computers. Nucleic Acids Res. 16: 1829-1836.

McCutcheon, J.P. and Eddy, S.R. 2003. Computational identification of non-coding RNAs in Saccharomyces cerevisiae by comparative genomics. Nucleic Acids Res. 31: 4119-4128.

Morrissey, J.P. and Tollervey, D. 1993. Yeast snR30 is a small nucleolar RNA required for $18 \mathrm{~S}$ rRNA synthesis. Mol. Cell. Biol. 13: $2469-2477$.

Ni, J., Tien, A.L., and Fournier, M.J. 1997. Small nucleolar RNAs direct site-specific synthesis of pseudouridine in ribosomal RNA. Cell 89: $565-573$.

Ofengand, J. and Bakin, A. 1997. Mapping to nucleotide resolution of pseudouridine residues in large subunit ribosomal RNAs from representative eukaryotes, prokaryotes, archaebacteria, mitochondria and chloroplasts. J. Mol. Biol. 266: 246-268.

Ofengand, J. and Fournier, M.J. 1998. The pseudouridine residues of rRNA: Number, location, biosynthesis, and function. In Modification and editing of RNA (eds. H. Grosjean and R. Benne), pp. 229-253. ASM Press, Washington DC.

Ofengand, J., Bakin, A., Wrzesinski, J., Nurse, K., and Lane, B.G. 1995. The pseudouridine residues of ribosomal RNA. Biochem. Cell. Biol. 73: 915-924.

Olivas, W.M., Muhlrad, D., and Parker, R. 1997. Analysis of the yeast genome: Identification of new non-coding and small ORFcontaining RNAs. Nucleic Acids Res. 25: 4619-4625.

Pogulis, R.J., Vallejo, A.N., and Pease, L.R. 1996. In vitro recombination and mutagenesis by overlap extension PCR. Methods Mol. Biol. 57: 167-176.

Puig, O., Caspary, F., Rigaut, G., Rutz, B., Bouveret, E., BragadoNilsson, E., Wilm, M., and Seraphin, B. 2001. The tandem affinity purification (TAP) method: A general procedure of protein complex purification. Methods 24: 218-229.

Raue, H.A., Klootwijk, J., and Musters, W. 1988. Evolutionary conservation of structure and function of high molecular weight ribosomal RNA. Prog. Biophys. Mol. Biol. 51: 77-129.

Saveanu, C., Bienvenu, D., Namane, A., Gleizes, P.E., Gas, N., Jacquier, A., and Fromont-Racine, M. 2001. Nog2p, a putative GTPase associated with pre-60S subunits and required for late 60S maturation steps. EMBO J. 20: 6475-6484.

Schattner, P., Decatur, W.A., Davis, C.A., Ares Jr., M., Fournier, M.J., and Lowe, T.M. 2004. Genome-wide searching for pseudouridylation guide snoRNAs: Analysis of the Saccharomyces cerevisiae genome. Nucleic Acids Res. 32: 4281-4296.

Tollervey, D. 1987. A yeast small nuclear RNA is required for normal processing of pre-ribosomal RNA. EMBO J. 6: 4169-4175.

Walter, A.E., Turner, D.H., Kim, J., Lyttle, M.H., Muller, P., Mathews, D.H., and Zuker, M. 1994. Coaxial stacking of helixes enhances binding of oligoribonucleotides and improves predictions of RNA folding. Proc. Natl. Acad. Sci. 91: 9218-9222. 

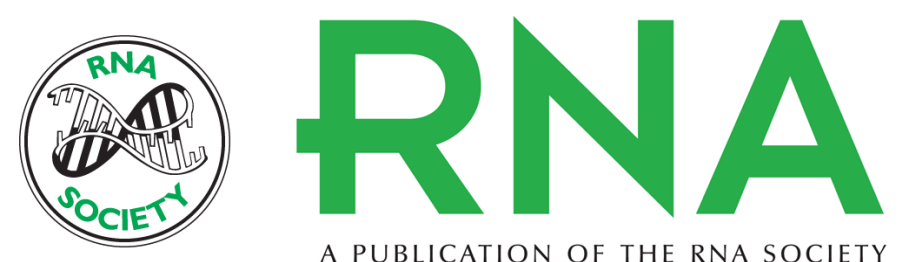

A PUBLICATION OF THE RNA SOCIETY

\section{The complete set of H/ACA snoRNAs that guide rRNA pseudouridylations in Saccharomyces cerevisiae}

CLAIRE TORCHET, GWENAËL BADIS, FRÉDÉRIC DEVAUX, et al.

RNA 2005 11: 928-938

References This article cites 33 articles, 8 of which can be accessed free at:

http://rnajournal.cshlp.org/content/11/6/928.full.html\#ref-list-1

License

Email Alerting Receive free email alerts when new articles cite this article - sign up in the box at the Service top right corner of the article or click here. 